\title{
Un catalyseur de changement
}

Parution anticipée. Publié sur www.jamc.ca le 24 février 2006.

$\mathrm{L}$ e rédacteur en chef du Journal, le $\mathrm{D}^{\mathrm{r}}$ John Hoey, et la rédactrice adjointe principale, Anne Marie Todkill, ont été congédiés le 20 février 2006. Leur départ porte un coup au $J A M C$, aux médecins du Canada, ainsi qu'aux milieux scientifiques et médicaux internationaux.

Le $\mathrm{D}^{\mathrm{r}}$ Hoey et $\mathrm{M}^{\mathrm{me}}$ Todkill sont arrivés au Journal en 1996 et 1994 respectivement. Ces deux rédacteurs de calibre mondial au talent immense sont voués à l'excellence et à l'intégrité en recherche scientifique et dans les reportages d'actualité, et déterminés à tenir compte du contexte social plus général de la médecine. Grâce à leurs efforts, le JAMC est devenu un journal médical international de premier plan. Son facteur d'impact a triplé pour atteindre 5,9, et jamc.ca, lancé pendant leur mandat, affiche maintenant plus de 250000 de pages visualisées par mois.

Le $\mathrm{D}^{\mathrm{r}}$ Hoey est un interniste, épidémiologiste et médecin spécialisé en santé publique respecté. Son amabilité, son imperturbabilité et son intelligence étaient d'excellentes qualités pour le rôle qu'il a joué à la barre du JAMC. $\mathrm{M}^{\text {me }}$ Todkill a fondé la chronique Côté cœur - section sur les arts et les lettres consacrée à la créativité de la médecine, à sa réflexion et à son visage parfois caché - et a dirigé la chronique Query, toutes deux bien accueillies par nos lecteurs. Ses questions pénétrantes, sa logique, sa maîtrise de la langue anglaise et ses conseils judicieux nous manqueront.

Sous la direction du $\mathrm{D}^{\mathrm{r}}$ Hoey et de $\mathrm{M}^{\mathrm{me}}$ Todkill, le Journal a abordé des sujets importants dans ses éditoriaux de premier plan sur la médecine et les soins de santé. Ils n'ont pas eu peur des questions controversées comme la décriminalisation de la marijuana, les effectifs des services d'urgence au Québec, l'avortement, l'assurance-maladie, la contraception d'urgence, la privatisation des soins de santé et l'indépendance rédactionnelle. Ils ont défendu fermement leurs principes et catalysé des changements. Que l'on accepte ou non leurs éditoriaux, ils ont indéniablement enrichi le discours de la médecine.

Nous reconnaissons que les propriétaires de tout journal ont la prérogative d'embaucher et de congédier ses rédacteurs. Les rédacteurs restants du JAMC protestent toutefois contre cette récente décision qu'ils n'acceptent pas. Nous demeurons en poste parce que nous croyons qu'il est crucial de n défendre les réalisations du $\mathrm{D}^{\mathrm{r}}$ Hoey et de $\mathrm{M}^{\mathrm{me}}$ Todkill et de les faire fructifier. Le Journal est devenu une importante tribune de discussion et de diffusion de documents de recherche originaux de grande qualité, d'articles de critique et de nouvelles qui aident à guider la pratique de la médecine. Il ne faut pas la mettre en danger.

Sur le plan légal, Holding AMC est propriétaire du Journal qui, dans un sens plus général, appartient toutefois aux patients, aux cliniciens, aux auteurs et aux lecteurs. Conformément aux efforts récents du $\mathrm{D}^{\mathrm{r}}$ Hoey et de $\mathrm{M}^{\mathrm{me}}$ Todkill ${ }^{1}$, nous nous sentons tenus envers la communauté de demander que l'on prenne des mesures qui garantiront une fois pour toutes l'indépendance rédactionnelle du JAMC. Le JAMC ne pourra demeurer un journal médical de premier plan et de réputation internationale que si ses rédacteurs sont libres de prendre des décisions rédactionnelles sans ingérence.

Quant à nous - afin d'aider à régler rapidement cette question - nous avons rédigé une proposition décrivant notre vision de l'indépendance du JAMC. Les commentaires de l'éditeur, du Comité de surveillance du Journal (CSJ) et de l'AMC seront les bienvenus.

Entre-temps, le départ de nos deux collègues, chefs de file et amis, nous attriste profondément.

Quant à nous, afin de régler rapidement la question, nous avons rédigé une proposition décrivant notre vision d'un $J A M C$ indépendant. Les commentaires du Conseil de rédaction, de l'éditeur, du CSJ et de l'AMC seront les bienvenus.

Nous remercions les membres de notre Conseil de rédaction de leur immense appui et nous les consulterons pour continuer à bénéficier de leurs conseils. Nous sommes reconnaissants aux membres du groupe consultatif à qui l'on a demandé de revoir l'autonomie rédactionnelle du JAMC et sa structure de gouvernance. Leur rapport est inclus dans ce numéro du Journal. Nous y incluons aussi une déclaration des présidents du Conseil d'administration de l'AMC et du CSJ au sujet de l'autonomie rédactionnelle.

\section{Stephen Choi}

Rédacteur en chef intérimaire

Ken Flegel

Rédacteur associé

Claire Kendall

Rédactrice associée

Anita Palepu

Rédactrice associée

Eric Wooltorton

Rédacteur associé

Sally Murray

Stagiaire postdoctorale en rédaction

Nick Barrowman

Consultant en statistique

$J A M C$

\section{RÉFÉRENCE}

I. L'autonomie de la rédaction du JAMC [éditorial]. JAMC 2006;174(I):II. 